\section{Lipidmanagement mit PCSK9-Inhibitoren}

Die Entdeckung des PCSK9 markiert einen Meilenstein in der Lipidforschung. Mit den PCSK9-Antikörpern steht jetzt ein sehr effektives Therapiekonzept für die LDL-Cholesterinsenkung zur Verfügung, das für Risikopatienten im Hinblick auf das kardiovaskuläre Risiko einen wesentlichen Fortschritt verspricht.

D as LDL-Cholesterin ist nicht nur ein Risikoindikator, sondern ein entscheidender Risikofaktor für die Arteriosklerose und somit die KHK. „Durch eine LDL-C-Senkung von $1 \mathrm{mmol} / \mathrm{l}$ kann das Risiko für ein schweres kardiovaskuläres Ereignis um circa 20 Prozent gesenkt werden", berichtete Prof. Winfried März, Mannheim. Und bei Senkung des LDLs $<50 \mathrm{mg} / \mathrm{dl}$ beträgt nach einer großen Metaanalyse das Einjahresrisiko für ein kardiovaskuläres Ereignis weniger als 5\% im Vergleich zu 16,5\% bei einem LDL zwischen 75 und $100 \mathrm{mg} / \mathrm{dl}$.

„Daher gilt für das LDL-C: The lower the better", so März. Bei Personen mit genetisch determiniertem niedrigen LDL-Wert werde durch ein um $1 \mathrm{mmol} / \mathrm{l}$ niedrigeres LDL-C das kardiovaskuläre Risiko sogar um über 70\% reduziert. Dies unterstreiche die Notwendigkeit einer frühen LDL-Senkung.

Die bisherige Standardtherapie zur LDL-Senkung sind Statine. „Doch mit einer solchen Substanz kann der LDLZielwert $<70 \mathrm{mg} / \mathrm{dl}$ meist auch mit höheren Dosen nicht erreicht werden, vor allem bei Patienten mit familiärer Hypercholesterinämie“, sagte Prof. Wolfgang Koenig, München. Für diese Patienten seien die PCSK9-Inhibitoren wie Alirocumab und Evolocumab ein wesentlicher Fortschritt. Nach vorliegenden Studiendaten könne mit diesen Substanzen der LDL-Wert um ca. 60\% gesenkt werden, eine Dimension die bis vor kurzem kaum vorstellbar gewesen sei.

\section{Von der Genetik zum Target}

PCSK9 ist ein wichtiges Regulatorprotein, denn es moduliert das Recycling der hepatischen LDL-Rezeptoren. PCSK9 ist eine Serinprotease, die in der Leber gebildet und ins Plasma sezerniert wird. Sie bindet an LDL-Rezeptoren auf der Leberzelloberfläche. Der entstandene Komplex wird von der Leberzelle aufgenommen und in Lysosomen abgebaut, d.h. das übliche Recycling des Rezeptors wird verhindert. Folge ist, dass die Menge an LDL-Rezeptoren abnimmt und das LDL-C im Blut ansteigt. Dagegen führt eine Hemmung von PCSK9 zu einem Anstieg der Rezeptoren und somit zu einer LDL-Senkung. „Die Entdeckung von PCSK9 2003 war ein wichtiger Meilenstein in der Lipidforschung", so Koenig.

Ausgangspunkt waren Beobachtungen bei Menschen mit einer „Loss-ofFunction"-Mutation, die ein sehr niedriges LDL haben und folglich ein sehr niedriges kardiovaskuläres Risiko. Umgekehrt gibt es „Gain-of-Function“-Mutationen mit hohen LDL-Werten und erhöhtem KHK-Risiko, die sich klinisch als familiäre Hypercholesterinämie manifestieren. „Antikörper gegen PCSK9 sind ein attraktives neues Target zur Therapie der Hypercholesterinämie“, so Koenig. Gerade Patienten mit hoher Plaquelast dürften von einer aggressiven LDL-Senkung profitieren; in Studien habe man mittels intravaskulärem Ultraschall nachgewiesen, dass eine starke LDL-Senkung zu einer Plaqueregression führe. Erste Daten sprechen dafür, dass sich die starke LDL-Senkung sogar günstig auf die kardiovaskuläre Ereignisrate auswirkt. Ergebnisse von Endpunktstudien liegen aber noch nicht vor.

\section{Neue Lipid-Dogmen}

Die derzeit komplex anmutenden und Empfehlungen für die LDL-C-Senkung dürften nach Meinung von März in absehbarer Zeit Vergangenheit sein. In Zukunft sollten angesichts der neuen Therapiemöglichkeiten ganz einfache Regeln für das Lipidmanagement gelten: -Je niedriger, umso besser! - Je früher, umso besser!

_Es gibt kein „zu niedriges Cholesterin“! _Es gibt nur einen Zielwert: $<70 \mathrm{mg} / \mathrm{dl}$. - Mit PCSK9-Inhibitoren ist er erreichbar. _Die Atherosklerose kann durch eine aggressive Cholesterinsenkung ausgerottet werden.

(sti) auch teils widersprüchlichen Leitlinien-

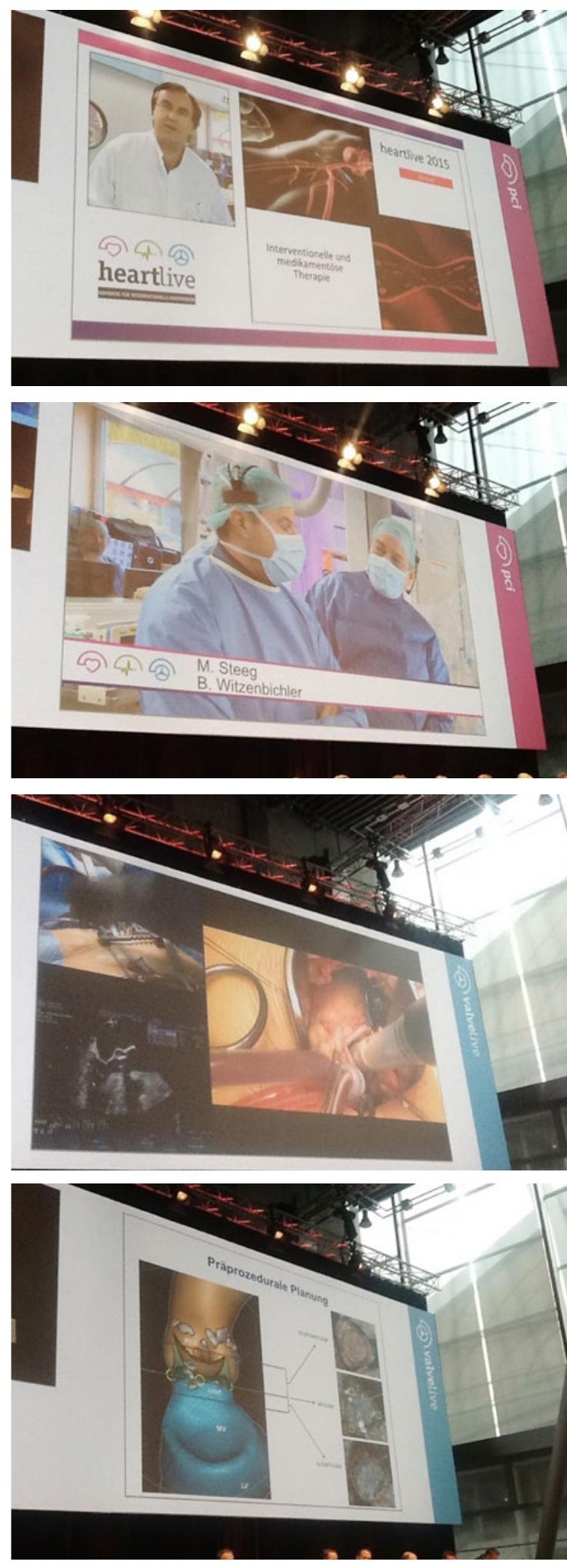

Ob PCl, minimalinvasive Mitralklappenrekonstruktion oder TAVI - an technischen Herausforderungen war auf dem Heartlive-Kongress in Stuttgart einiges geboten. 